\title{
The balance between shocks and AGN photoionization in radio sources and its relation to the radio size
}

\author{
E. Moy ${ }^{1,2}$ and B. Rocca-Volmerange ${ }^{1,3}$ \\ 1 Institut d'Astrophysique de Paris, 98bis boulevard Arago, 75014 Paris, France \\ 2 Max-Planck-Institut für extraterrestrische Physik, Postfach 1312, 85741 Garching, Germany \\ 3 Institut d'Astrophysique Spatiale, Bât. 121, Université Paris XI, 91405 Orsay, France \\ Received 27 April 2001 / Accepted 26 November 2001
}

\begin{abstract}
We have analyzed the ultraviolet and optical emission line ratios of a large sample of extragalactic radio sources (QSOs and radio galaxies), with the help of models combining AGN photoionization and shocks. The results strongly suggest that the two ionizing mechanisms frequently coexist. The model sequences obtained by varying the balance between shocks and AGN photoionization account for most emission line data in the 12 line ratio diagrams we have considered. In the frequently used diagrams involving [OIII] $\lambda 5007 / \mathrm{H} \beta$, $[\mathrm{OI}] \lambda 6300 / \mathrm{H} \alpha,[\mathrm{NII}] \lambda 6584 / \mathrm{H} \alpha$ and $[\mathrm{SII}] \lambda \lambda 6716,6731$ (Veilleux \& Osterbrock 1987), the effect of varying the shock-photoionization balance mimics a variation of the ionization parameter $(U)$ in traditional photoionization sequences. In most of the remaining diagrams, such as $[\mathrm{OI}] \lambda 6300 /[\mathrm{OIII}] \lambda 5007$ vs. $[\mathrm{OIII}] \lambda 4363 /[\mathrm{OIII}] \lambda 5007$, $[\mathrm{OIII}] \lambda 5007 / \mathrm{H} \beta$ vs. $[\mathrm{OIII}] \lambda 4363 /[\mathrm{OIII}] \lambda 5007$ and CIII $] \lambda 1909 /[\mathrm{CII}] \lambda 2326$ vs. CIV $\lambda 1549 /[\mathrm{CII}] \lambda 2326$, the data can only be accounted for if both photoionization and shocks contribute to the line fluxes. The coexistence of shocks and AGN photoionization also provides an explanation for the most extreme objects in the NV $\lambda 1240 / \mathrm{HeII} \lambda 1640$ vs. NV $\lambda 1240 /$ CIV $\lambda 1549$ diagram without requiring largely super-solar metallicities. In addition, we show that there is a relationship between the $[\mathrm{OII}] \lambda 3727 /[\mathrm{OIII}] \lambda 5007$ ratio (i.e., the ionization level of the gas) and the radio size in radio galaxies. This strongly supports the hypothesis that the most compact $(\leq 2 \mathrm{kpc})$ and the largest $(\geq 150 \mathrm{kpc})$ sources are dominated by photoionization, while intermediate-sized radio galaxies are dominated by shocks. We briefly discuss the possible origin of the relation between the shock-ionization balance and the radio size.
\end{abstract}

Key words. line: formation - galaxies: active - galaxies: evolution - galaxies: ISM - quasars: emission lines

\section{Introduction}

One of the most debated questions about the interaction between active galactic nuclei (AGN) and their environment is the nature of the ionizing source of the extended structures of ionized gas detected in powerful radio galaxies (Spinrad \& Djorgovski 1984; Tadhunter et al. 1988; Baum et al. 1988) and QSOs (Hes et al. 1996). The emission line spectra of these regions impose the strongest constraints on this issue. Initial studies of extended line emission in radio sources showed that photoionization models provide an acceptable fit of emission line ratios in the optical (Robinson et al. 1987; Saunders et al. 1989; Prieto et al. 1993) and the UV (Villar-Martín et al. 1997), in good agreement with the "unified scheme" (e.g. Antonucci 1993).

However, some properties of the extended emission cannot be accounted for by the photoionization hypothesis. Some AGN have a spectrum dominated by low ionization lines (e.g. Koski \& Osterbrock 1976; Durret 1990), or show [OIII $\lambda 44363 /[\mathrm{OIII}] \lambda 5007$ ratios

Send offprint requests to: E. Moy, e-mail: moy@mpe.mpg.de indicative of high electron temperatures $\left(T_{\mathrm{e}} \sim 20000 \mathrm{~K}\right.$; Ferland \& Osterbrock 1987). These two properties are more reminiscent of ionizing shocks. Shocks can be produced, for example, by the interactions of the radio ejecta with the interstellar medium (ISM), as suggested by the spatial coincidence between radio and line emissions in radio sources (Baum \& Heckman 1989; McCarthy et al. 1995; Ridgway \& Stockton 1997; Lehnert et al. 1999). The disturbed kinematics of radio galaxies are also indicative of the influence of shocks in the ionizing process (VillarMartín et al. 1999a). With the help of theoretical models (Dopita \& Sutherland 1996), evidence of ionizing shocks has been found in an increasing number of AGN from the analysis of their line ratios (Sutherland et al. 1993; Clark et al. 1998; Koekemoer \& Bicknell 1998; Villar-Martín et al. 1999c).

Despite their success, however, shock models remain on average less efficient than photoionization models in explaining the emission line properties of AGN, especially in the UV (Villar-Martín et al. 1997; De Breuck et al. 2000). Therefore, shocks should probably not be regarded as an alternative to AGN photoionization, but more likely as an 
additional ionization source that may be dominant only under some circumstances. In this paper we investigate the possibility that shocks induced by the radio ejecta and AGN photoionization coexist in QSOs and radio galaxies.

To test this hypothesis, we systematically compare observed emission line ratios, both in the UV and in the optical, with models combining the two ionization processes in varying proportions. The shock models are computed with MAPPINGSIII (Dopita \& Sutherland 1996) and the photoionization models with CLOUDY (Ferland 1996). Our approach implicitly assumes that shocks and photoionization don't occur simultaneously in individual clouds. This is a reasonable approximation in the case of jet-induced shocks located near radio hot spots (Clark et al. 1998; Villar-Martín et al. 1999b). At such distances from the nucleus, the AGN radiation is weak due to geometrical dilution, and the ionization by shocks should be dominant. Conversely, clouds lying closer to the AGN should be mainly photoionized. However, our models probably don't apply in the case of shocks induced by inflows or outflows (see Contini \& Aldrovandi 1983; Contini \& Aldrovandi 1986; Aldrovandi \& Contini 1984, 1985; Contini \& ViegasAldrovandi 1987; Viegas-Aldrovandi \& Contini 1989).

We also discuss in detail the relationship between the radio size and the role of shocks in the ionizing process. The present work confirms the conclusion of Best et al. (2000b) that large radio sources are dominated by AGN photoionization. Here we show that compact sources are also dominated by AGN photoionization. Shocks play a major role only in some of the intermediate-sized radio sources.

Section 2 presents our modeling of the ionizing processes with the help of CLOUDY and MAPPINGSIII. The data set used for the comparison with the model predictions is described in Sect. 3. The results of this comparison are presented in Sect. 4, with a particular emphasis on the NV $\lambda 1240 / \mathrm{HeII} \lambda 1640$ vs. NV $\lambda 1240 / \mathrm{CIV} \lambda 1549$ diagram, because of its importance as a metallicity diagnostic. In Sect. 5, we discuss the evidence for ionizing shocks in radio sources, and investigate the possible origin of the link between the shock-photoionization balance and the radio size. We summarize our conclusions in Sect. 6 .

\section{Modeling the ionizing processes}

\subsection{Photoionization by the central AGN}

AGN photoionization models (hereafter A) are computed with CLOUDY (Ferland 1996). The emission line spectra of photoionized materials depend on the slope of the ionizing continuum, on the ionization parameter $U^{1}$, on the gas density and on its metallicity. The ionizing continuum follows a power law $\left(\Phi_{\nu} \propto \nu^{\alpha}\right)$ truncated at 0.01 and 20 Ryd.

Two values of the spectral index are considered: $\alpha=-1$, in agreement with the analysis of UV line

${ }^{1}$ Defined as $U=F^{\mathrm{H}_{0}} / n_{\mathrm{H}} c$, where $F^{\mathrm{H}_{0}}$ is the ionizing flux and $n_{\mathrm{H}}$ the hydrogen density. ratios in high- $z$ radio galaxies (Villar-Martín et al. 1997), and $\alpha=-1.5$, as suggested by optical line ratios in low- $z$ sources (Robinson et al. 1987). The ionization parameter varies between $\log U=-4$ and $\log U=-1$. We consider two values for the hydrogen density: $n_{\mathrm{H}}=10^{2} \mathrm{~cm}^{-3}$ and $n_{\mathrm{H}}=10^{6} \mathrm{~cm}^{-3}$. The first value is typical of extended emission line regions (McCarthy et al. 1990). The second one accounts for a possible contamination of UV lines by denser regions, as suggested by Villar-Martín et al. (1999b). Two different metallicities, $Z=Z_{\odot}$ and $Z=$ $1.5 Z_{\odot}$, are considered. The abundances are as in Moy et al. (2001), except for nitrogen: in the present work the evolution of the $\mathrm{N} / \mathrm{O}$ ratio with $Z$ follows the empirical relation proposed by Bresolin et al. (1999).

\section{2. lonizing shocks}

We use MAPPINGSIII (Dopita \& Sutherland 1996) to compute the emission line spectra emitted by shocked materials. The precursor density is $1 \mathrm{~cm}^{-3}$, as in Dopita \& Sutherland (1995). We considered shock velocities $v$ between 100 and $1000 \mathrm{~km} \mathrm{~s}^{-1}$. The magnetic parameter $B / \sqrt{(n)}$ is fixed to 3 in all calculations. This value corresponds to equipartition between thermal and magnetic pressures (Dopita \& Sutherland 1996). For the sake of consistency, metallicities $\left(Z_{\odot}\right.$ and $\left.1.5 Z_{\odot}\right)$ and chemical abundances are exactly those adopted for photoionization calculations. We consider both pure shock (hereafter S) and shock+precursor (hereafter SP) models.

The upward spectrum produced by a $v=1000 \mathrm{~km} \mathrm{~s}^{-1}$ shock is too hard to be fully absorbed by the precursor region. To construct an appropriate SP model for $v=$ $1000 \mathrm{~km} \mathrm{~s}^{-1}$, the precursor model is therefore truncated at $F_{\mathrm{HII}} \sim 0.7$, where $F_{\mathrm{HII}}$ is the fraction of ionized hydrogen, so that it is "matter-bounded" (Viegas \& Prieto 1992).

\subsection{Combining photoionization and shock models}

A two-component model is obtained by combining the results of a shock model (characterized by the shock velocity $v$ ) and of a photoionization model (characterized by the ionization parameter $U$ ). Only models with similar metallicities are combined in this way. The total flux $F_{i}$ in a given line $i$ is computed as follows:

$$
F_{i}=A_{\mathrm{A} / \mathrm{S}} \times F_{i}^{v}+\left(1-A_{\mathrm{A} / \mathrm{S}}\right) \times F_{i}^{U} \quad A_{\mathrm{A} / \mathrm{S}} \in[0,1]
$$

where $F_{i}^{v}$ and $F_{i}^{U}$ are the fluxes predicted by the shock and photoionization models, respectively. By varying the $A_{\mathrm{A} / \mathrm{s}}$ parameter between 0 and 1 , we obtain full sequences between the "pure shock" and the "pure photoionization" cases.

\section{Data sources}

Our data compilation, listed in the Appendix (Table A.1), includes emission lines for 369 radio galaxies. 122 have a Fanaroff \& Riley (1974) classification (10 FR1s and 112 FR2s). The sample also includes 23 compact steep 

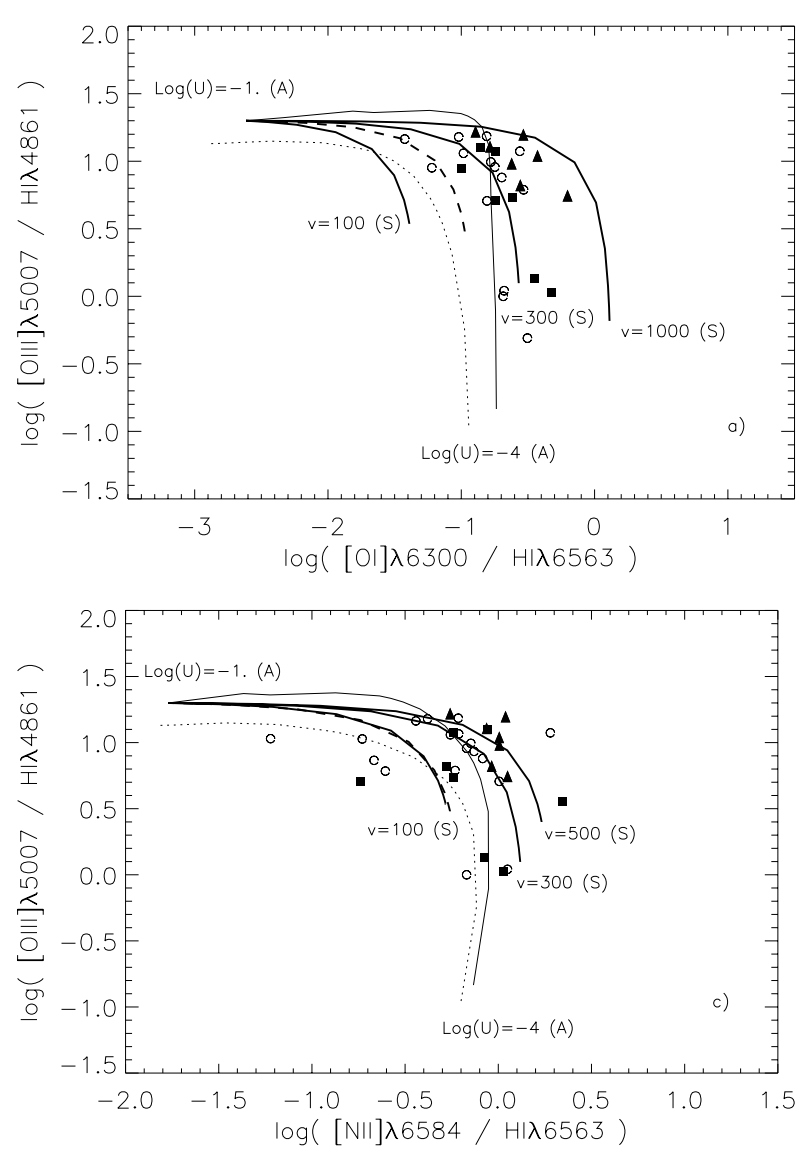

spectrum (CSS) objects and 59 QSOs. For objects with both narrow and broad lines, only the narrow component is considered. We classify the observations according to their spatial coverage: nuclear (including only the central regions), extended (including only the off-nuclear regions), and spatially integrated (covering both the nuclear and extended regions). We classified the observations of Simpson et al. (1996) as "spatially integrated" in spite of their limited spatial coverage, since they include at least part of the extended emission. The radio sizes are estimated from the apparent largest angular sizes (LAS), assuming $\Omega_{0}=1$, $\Lambda_{0}=0$ and $H_{0}=50 \mathrm{~km} \mathrm{~s}^{-1} \mathrm{Mpc}^{-1}$. The source papers for the LAS are listed in Col. 3 of Table A1. For sources from Morganti et al. (1993), the LAS were directly measured from the radio images.

\section{Diagnostic diagrams with combined models}

Figures 1 to 6 present the comparisons between the model predictions and our compilation of line ratios. Since large ranges of ionization parameter and shock velocity are considered, the number of $A_{\mathrm{A} / \mathrm{S}}$ sequences is very high. For the sake of clarity, only a few sequences, characterized by the parameter pair $(\log U, v)$, are plotted on each figure. These are $(\log U, v)=(-1,300),(-1,100)$, and $(-1,1000)$ for $A_{\mathrm{A} / \mathrm{S}}$ sequences combining one photoionization model with one pure shock model, hereafter $\mathrm{A}+\mathrm{S}$ sequences (thick solid lines). The first of these sequences provides a good fit to the data in many optical diagrams and is therefore regarded as the "reference" sequence. The

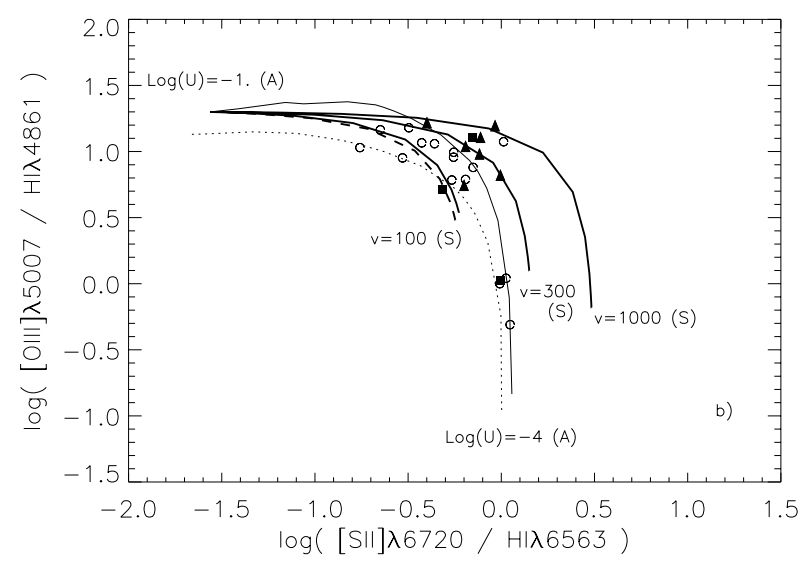

Fig. 1. Comparison between the predictions of two component A $+\mathrm{S}$ models (thick solid lines) and observational data in the three diagrams of Veilleux \& Osterbrock (1987). The metallicity is solar, and the density $n_{\mathrm{H}}$ is equal to $100 \mathrm{~cm}^{-3}$. The values of $\log U$ and $v$ are indicated on the plot. Two photoionization model sequences $(-4 \leq \log U \leq-1)$, with spectral index $\alpha=-1$ (thin solid line) and $\alpha=-1.5$ (dotted line), are shown for comparison. An example of an A+SP model sequence with $\log U=-1$ and $v=300 \mathrm{~km} \mathrm{~s}^{-1}$ is also plotted (dashed line). Filled squares indicate emission line ratios for spatially integrated (nuclear + extended) emission line regions, filled triangles for nuclear regions only, and open circles for extended regions only. Note the similarity between the $\mathrm{A}+\mathrm{S}$ sequences and the pure photoionization sequences.

latter two sequences usually define the borders of the area covered by two-component models.

One example sequence which includes the emission from a precursor (hereafter $\mathrm{A}+\mathrm{SP}$ sequence), with $(\log U, v)=(-1,300)$, is also plotted (dashed line). A "classical" AGN photoionization model sequence with spectral index $\alpha=-1$ and $-4 \leq \log U \leq-1$ is shown for comparison (thin line). If not specified, the metallicity is always solar, and the hydrogen density $n_{\mathrm{H}}$ is always $100 \mathrm{~cm}^{-3}$. Some diagrams also plot an additional model sequence (dotted line) with different parameters, whose values depend on the line ratio under consideration (see below).

\subsection{Optical line ratios}

\subsubsection{Veilleux \& Osterbrock (1987) diagrams}

Figure 1 shows the comparisons between the various models and our compilation of data in the three diagnostic diagrams of Veilleux \& Osterbrock (1987): $[\mathrm{OIII}] \lambda 5007 / \mathrm{H} \beta$ vs. $[\mathrm{OI}] \lambda 6300 / \mathrm{H} \alpha$ (Fig. 1a), $[\mathrm{OIII}] \lambda 5007 / \mathrm{H} \beta \quad$ vs. $[\mathrm{SII}] \lambda 6720 / \mathrm{H} \alpha \quad$ (Fig. 1b) and $[\mathrm{OIII}] \lambda 5007 / \mathrm{H} \beta$ vs. $[\mathrm{NII}] \lambda 6584 / \mathrm{H} \alpha$ (Fig. 1c). On each graph, the $\mathrm{A}+\mathrm{S}$ sequences (thick solid lines) are very similar to the pure AGN photoionization sequence (thin line). This result demonstrates that a variation of the balance between shocks and AGN photoionization mimics a variation of the ionization parameter $U$, which has long 

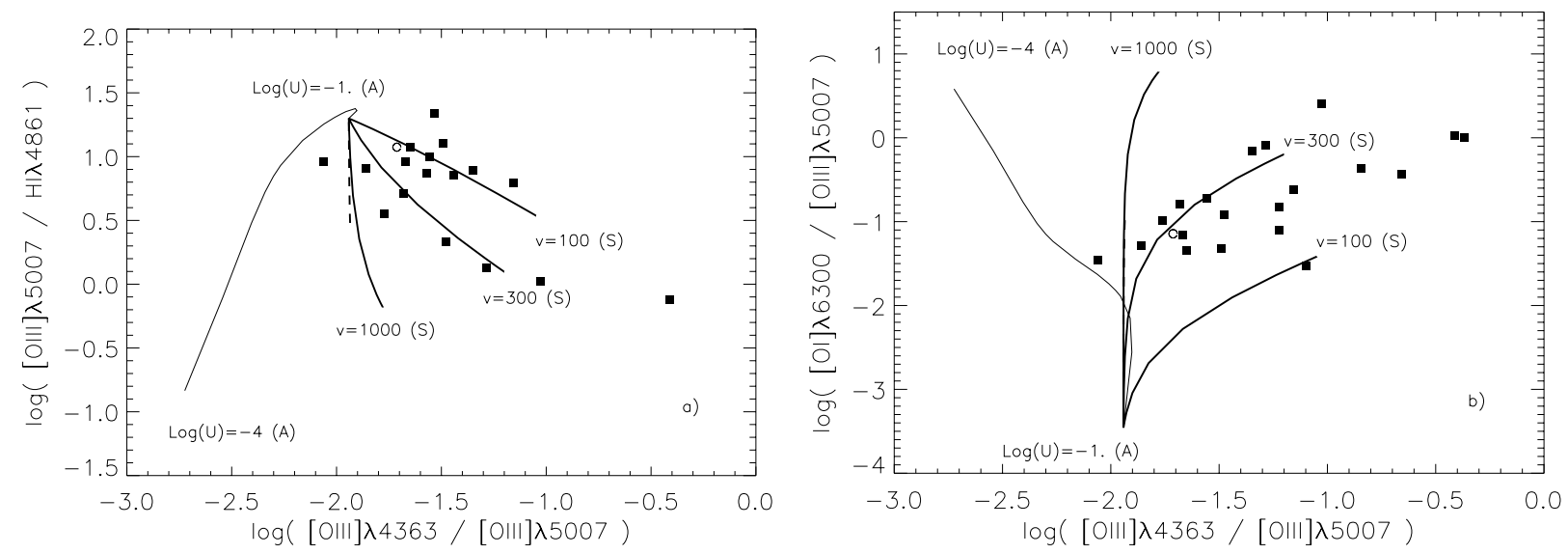

Fig. 2. $[\mathrm{OIII}] \lambda 5007 / \mathrm{H} \beta$ and $[\mathrm{OI}] \lambda 6300 /[\mathrm{OIII}] \lambda 5007$ vs. $[\mathrm{OIII}] \lambda 4363 /[\mathrm{OIII}] \lambda 5007$. Symbols as in Fig. 1. The photoionization sequence with $\alpha=-1.5$ is not plotted. Remaining curves as in Fig. 1: thick solid lines are A+S models; thin solid line are pure photoionization models $(-4 \leq \log U \leq-1)$, with spectral index $\alpha=-1$; dashed line are A+SP models. The data trends and dispersions are compatible with $\mathrm{A}+\mathrm{S}$ models. The A+SP sequence (dashed line) agrees almost exactly with the $v=1000 \mathrm{~km} \mathrm{~s}$ $\mathrm{A}+\mathrm{S}$ sequence. Pure photoionization models are clearly excluded.

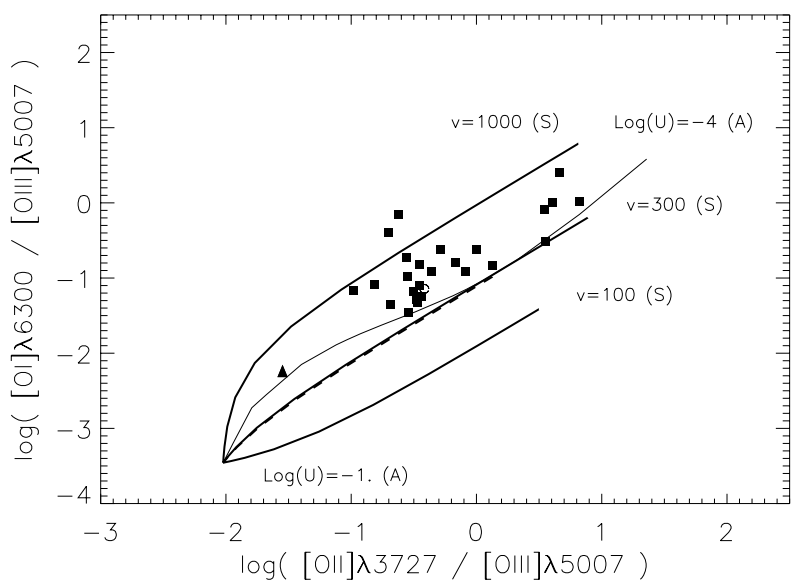

Fig. 3. $[\mathrm{OI}] \lambda 6300 /[\mathrm{OIII}] \lambda 5007$ vs. $[\mathrm{OII}] \lambda 3727 /[\mathrm{OIII}] \lambda 5007$. Symbols as in Fig. 1, and curves as in Fig. 2. The A+SP (dashed line) sequence is superimposed to the $v=300 \mathrm{~km} \mathrm{~s}^{-1}$ $\mathrm{A}+\mathrm{S}$ sequence. As in Fig. 1, the $\mathrm{A}+\mathrm{S}$ sequences (thick lines) are very similar to the pure photoionization sequence (thin line), but with a larger range of $[\mathrm{OI}] \lambda 6300 /[\mathrm{OIII}] \lambda 5007$.

been the traditional way of interpreting optical line ratio diagrams.

The $\alpha=-1$ photoionization models (thin solid line) underpredict the observed $[\mathrm{OI}] \lambda 6300 / \mathrm{H} \alpha,[\mathrm{SII}] \lambda 6720 / \mathrm{H} \alpha$ and $[\mathrm{NII}] \lambda 6584 / \mathrm{H} \alpha$ ratios, sometimes by as much as 0.5 dex. In the case of $\alpha=-1.5$ (dotted line), the situation is even worse, due to the lack of high-energy photons and the subsequent reduction of the "partially ionized zone". In order to reconcile pure photoionization models with the data, one could in principle assume either a lower metallicity (see Ferland \& Netzer 1983) or a higher density. However, the first hypothesis does not solve the discrepancy for $[\mathrm{NII}] \lambda 6584 / \mathrm{H} \alpha$, as already emphasized by Ferland \& Netzer (1983) and Veilleux \& Osterbrock (1987). The second hypothesis is ruled out by the high $[\mathrm{SII}] \lambda 6720 / \mathrm{H} \alpha$ ratios observed in our sample. Due to collisional deexcitation, the sulphur lines would be weaker than observed if densities were higher than $n_{\mathrm{H}}=100 \mathrm{~cm}^{-3}$. $\mathrm{A}+\mathrm{S}$ models (combining AGN photoionization and pure shocks; thick lines) account for most of the data. $\mathrm{A}+\mathrm{SP}$ models (combining AGN photoionization and shocks+precursors; dashed line) are acceptable as well. The sequence with $(\log U, v)=(-1,300)$ provides the best fit to the data on the three diagrams. The dispersion around this sequence, especially in $[\mathrm{OI}] \lambda 6300 / \mathrm{H} \alpha$ (Fig. 1a) and $[\mathrm{SII}] \lambda 6720 / \mathrm{H} \alpha$ (Fig. 1b), requires that the shock velocity varies between 100 and $1000 \mathrm{~km} \mathrm{~s}^{-1}$.

Most data points lie above $\log ([\mathrm{OIII}] \lambda 5007 / \mathrm{H} \beta)=0.5$, and only five show a low ionization level $(\log ([\mathrm{OIII}] \lambda 5007 / \mathrm{H} \beta) \sim 0$. $)$. These objects (or regions) are probably completely dominated by shocks. The fact that there is no nuclear region in this group supports our initial hypothesis (Sect. 1) that shocks mainly occur far from the nucleus.

\subsubsection{The $[\mathrm{OIII}] \lambda 4363 /[\mathrm{OIII}] \lambda 5007$ ratio}

The so-called "temperature problem" has long been an outstanding question in the modeling of emission lines in AGN. Photoionization models predict too low $[\mathrm{OIII}] \lambda 4363 /[\mathrm{OIII}] \lambda 5007$ ratios (i.e., too low temperatures) compared to the observations. (e.g. Tadhunter et al. 1989). Dopita \& Sutherland (1995) showed that pure shock models are able to account for $\log ([\mathrm{OIII}] \lambda 4363 /[\mathrm{OIII}] \lambda 5007)$ as high as -1 . However, these models underpredict the ionization level of the gas and are unable to account for the $[\mathrm{OIII}] \lambda 5007 / \mathrm{H} \beta$ ratios in AGN (see Fig. 7 in Dopita \& Sutherland 1995).

Figure. 2 presents the temperature-sensitive $[\mathrm{OIII}] \lambda 4363 /[\mathrm{OIII}] \lambda 5007$ ratio vs. the two ionization levelsensitive $[\mathrm{OIII}] \lambda 5007 / \mathrm{H} \beta$ and $[\mathrm{OI}] \lambda 6300 /[\mathrm{OIII}] \lambda 5007$ ratios (Figs. 2a and 2b, respectively). From these plots it is clear that $\mathrm{A}+\mathrm{S}$ sequences solve the temperature problem. The bulk of the $[\mathrm{OIII}] \lambda 4363$ emission originates in shocks, while the [OIII] $\lambda 5007$ line is emitted mainly 

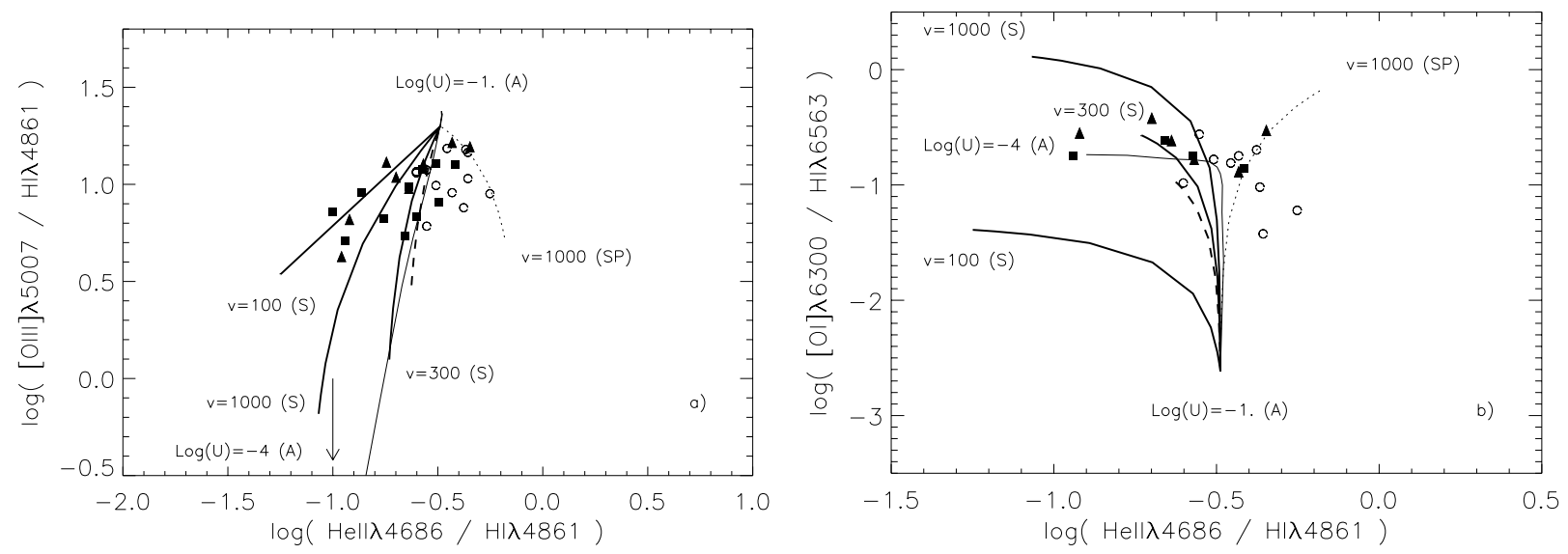

Fig. 4. $[\mathrm{OIII}] \lambda 5007 / \mathrm{H} \beta$ and $[\mathrm{OI}] \lambda 6300 / \mathrm{H} \alpha$ vs. HeII $\lambda 4686 / \mathrm{H} \beta$. Symbols as in Fig. 1, and curves as in Fig. 2 . (except for the dotted line; see below). Many extended regions (open circles) show high HeII $\lambda 4686 / \mathrm{H} \beta$ ratios. They can be accounted for by $\mathrm{A}+\mathrm{SP}$ models if the precursor is matter bounded (dotted line).

by the photoionized component. An alternative to the $\mathrm{A}+\mathrm{S}$ solution would be to increase the density, but we have shown in the previous section that a significant contamination of the optical lines by a high-density zone is unlikely.

The data distribution in the $[\mathrm{OI}] \lambda 6300 /[\mathrm{OIII}] \lambda 5007$ vs. [OIII $] \lambda 4363 /[\mathrm{OIII}] \lambda 5007$ (Fig. 2b) is striking. In the classical AGN photoionization hypothesis, one would expect an inverse correlation between these two ratios, since $[\mathrm{OIII}] \lambda 4363$ weakens faster than $[\mathrm{OIII}] \lambda 5007$ at low $U$ (thin line). On the contrary, we find that $[\mathrm{OI}] \lambda 6300 /[\mathrm{OIII}] \lambda 5007$ and $[\mathrm{OIII}] \lambda 4363 /[\mathrm{OIII}] \lambda 5007$ are correlated. A Spearman's rank test gives a correlation coefficient of 0.41 with a significance level of $\sim 5 \times 10^{-7}$. This means that objects showing the lowest ionization level also have the highest electronic temperature. As illustrated in Fig. 2b, this trend is qualitatively reproduced by $\mathrm{A}+\mathrm{S}$ models. Again, this result strongly suggests that the observational sequence from high-ionization to low-ionization radio sources is actually a sequence from photoionizationdominated to shock-dominated objects.

\subsection{3. [OI] $\lambda 6300 /[\mathrm{OIII}] \lambda 5007$ vs. [OII] $\lambda 3727 /[\mathrm{OIII}] \lambda 5007$}

This diagram involves two line ratios which are sensitive to the ionization level of the gas. They are frequently used in the literature as a diagnostic of the ionization source of the gas (e.g. Baldwin et al. 1981). Again, Fig. 3 illustrates:

- that a variation of the balance between shocks and AGN photoionization quantitatively mimics a variation of the ionization parameter $U$ in classical line ratio diagrams; and

- that $\mathrm{A}+\mathrm{S}$ and $\mathrm{A}+\mathrm{SP}$ models are better able than pure photoionization models to account for the data.

It is clear from this plot that there is a partial degeneracy between the ionization parameter of the photoionized component and the value of $A_{\mathrm{A} / \mathrm{S}}$, and that $\log U$ could be less than -1 in some objects. Even so, shocks are still needed to account for the $[\mathrm{OI}] \lambda 6300 / /[\mathrm{OIII}] \lambda 5007$.

Interestingly, shock models predict a relatively small range of $\log ([\mathrm{OII}] \lambda 3727 /[\mathrm{OIII}] \lambda 5007)$ which is systematically higher than the predictions of AGN photoionization models, in contrast to other line ratios highly influenced by shocks like $[\mathrm{OI}] \lambda 6300 / \mathrm{H} \alpha,[\mathrm{NII}] \lambda 6584 / \mathrm{H} \alpha$ and $[\mathrm{SII}] \lambda 6720 / \mathrm{H} \alpha$ (see Fig. 1). The $[\mathrm{OII}] \lambda 3727 /[\mathrm{OIII}] \lambda 5007$ ratio is therefore an unambiguous indicator of the presence of ionizing shocks inside a given object.

\subsubsection{The Hell $\lambda 4686 / \mathrm{H} \beta$ ratio}

It is well known that the $\mathrm{HeII} \lambda 4686 / \mathrm{H} \beta$ ratio cannot be accounted for by classical photoionization models. Figure 4 shows that $\mathrm{A}+\mathrm{S}$ and $\mathrm{A}+\mathrm{SP}$ models (thick lines) account for most of the data data in the $[\mathrm{OIII}] \lambda 5007 / \mathrm{H} \beta$ vs. $\mathrm{HeII} \lambda 4686 / \mathrm{H} \beta$ (Fig. 4a) and $[\mathrm{OI}] \lambda 6300 / \mathrm{H} \alpha$ vs. HeII $\lambda 4686 / \mathrm{H} \beta$ (Fig. 4b). However, these models still underpredict $\mathrm{HeII} \lambda 4686 / \mathrm{H} \beta$ by $\sim 0.5$ dex for a few data (open circles), especially in Fig. 4a.

Binette et al. (1996) showed that photoionized matterbounded (MB) clouds were one solution which could account for the highest $\mathrm{HeII} \lambda 4686 / \mathrm{H} \beta$ ratios observed in AGN. In this picture, however, MB clouds are necessarily located near the nucleus. This scenario is unlikely to work for our data since most of the problematic points correspond to "extended" regions. As an alternative, we argue that MB precursors may well explain the existence of off-nuclear regions with high $\mathrm{HeII} \lambda 4686 / \mathrm{H} \beta$ ratios. Precursors are likely to lie far from the nucleus if, as our hypothesis predicts, shocks occur mainly near the radio hot spots. Moreover, their ionization level is high, thanks to the proximity of their ionization source (Dopita $\&$ Sutherland 1996). Figure 4 plots the prediction of the $v=1000 \mathrm{~km} \mathrm{~s}^{-1} \mathrm{SP}$ model with a truncated precursor (see Sect. 2.2). We conclude that MB precursors, the existence of which is discussed in Sect. 5.1, are able to account for $\log (\operatorname{HeII} \lambda 4686 / \mathrm{H} \beta) \sim-0.3$ in extended regions. 

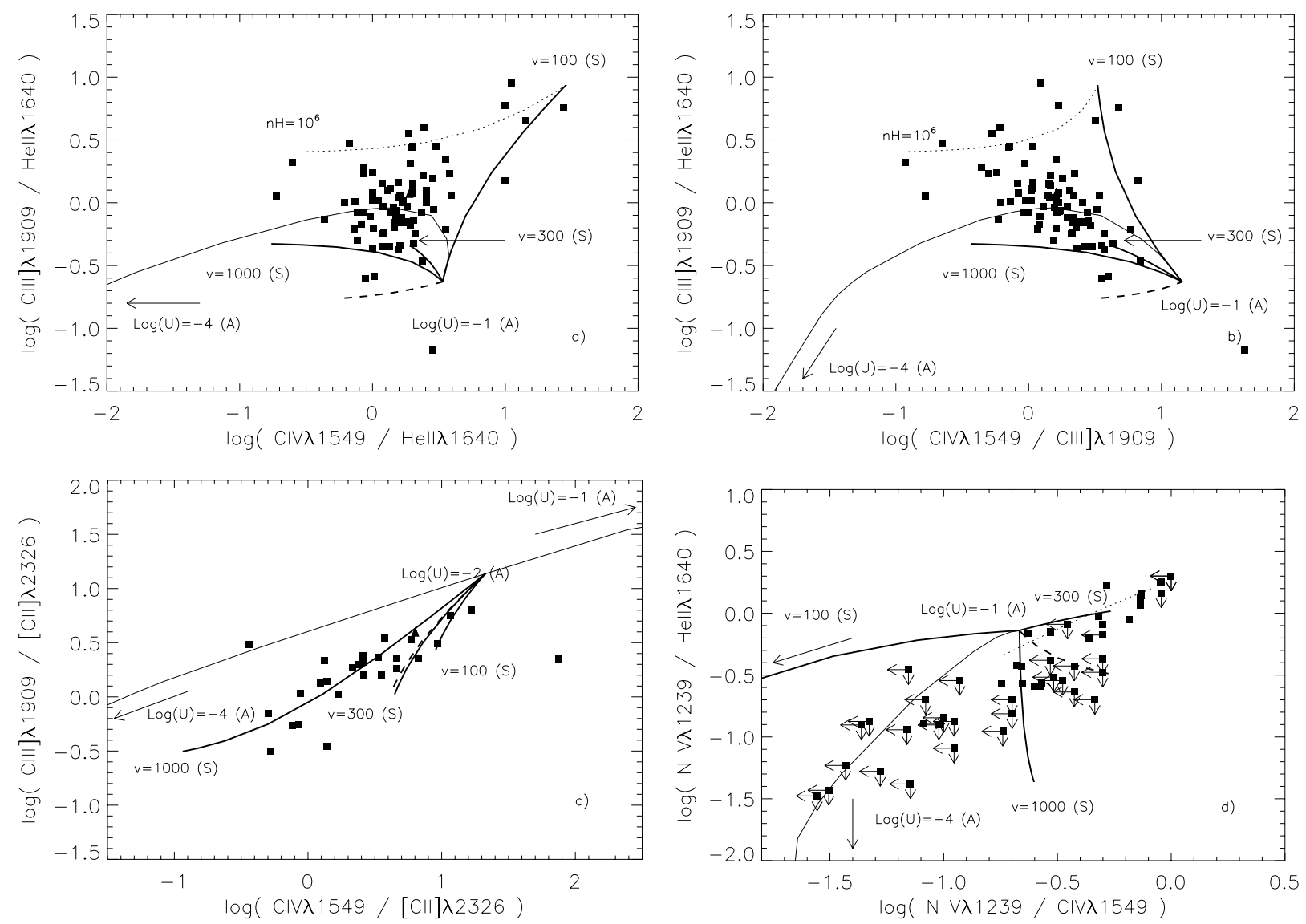

Fig. 5. Comparisons between the predictions of $\mathrm{A}+\mathrm{S}$ and $\mathrm{A}+\mathrm{SP}$ models and observational data for UV line ratios. Symbols as in Fig. 1. Thick solid curves, thin solid curves, and dashed curve are as in Fig. 2, except for Fig. 5c (see below). The dotted curve in Figs. 5a and 5b shows the $\mathrm{A}+\mathrm{S}$ sequence with $\log U=-3 v=100$, if the density of the photoionized component is $n_{\mathrm{H}}=10^{6} \mathrm{~cm}^{-3}$. The contamination of the emission line spectrum by the NLR is strongly suggested by these diagrams. In Fig. $5 \mathrm{c}, \log U=-1$ has been replaced by $\log U=-2$ in the $\mathrm{A}+\mathrm{S}$ sequence. In Fig. $5 \mathrm{~d}$, the dotted line shows the $\mathrm{A}+\mathrm{S}$ sequence with $\log U=-1$ and $v=300 \mathrm{~km} \mathrm{~s}^{-1}$ for $Z=1.5 Z_{\odot}$.

\subsection{UV line ratios}

We have shown in the previous section that the coexistence of shocks and photoionization in AGN is strongly favored by analysis of optical line ratios. The distribution of the data in many diagrams is incompatible with pure photoionization models, while the $\mathrm{A}+\mathrm{S}$ sequence with $\log U \sim-1$ and $v=300 \mathrm{~km} \mathrm{~s}^{-1}$ provides a good fit to the observations. The scattering of the data around this sequence is well accounted for by $\mathrm{A}+\mathrm{S}$ sequences with $100 \mathrm{~km} \mathrm{~s}^{-1} \leq v \leq 1000 \mathrm{~km} \mathrm{~s}^{-1}$. This result needs to be confirmed in the UV. However, such a study is difficult due to the possible contamination of the UV lines by the narrow line region (NLR), wich has densities of up to $n_{\mathrm{H}}=10^{6} \mathrm{~cm}^{-3}$ (Villar-Martín et al. 1999b). In what follows we show that the UV line fluxes observed in radio sources are compatible with the coexistence of AGN photoionization and shocks, if the contamination by the NLR is taken into account.

Figure 5 presents the predictions of the $\mathrm{A}+\mathrm{S}$ and A+SP models compared to the UV data. An A+S sequence with a high density $\left(n_{\mathrm{H}}=10^{6} \mathrm{~cm}^{-3}\right)$ photoionization component $(\log U=-3)$ is also shown for comparison (dotted line) on the diagrams involving density-sensitive line ratios. The data distributions are completely accounted for in the CIII $] \lambda 1909 / \mathrm{HeII} \lambda 1640$ vs. CIV $\lambda 1549 / \mathrm{HeII} \lambda 1640$ (Fig. 5a) and CIII] $\lambda 1909 / \mathrm{HeII} \lambda 1640$ vs. CIV $\lambda 1549 / \mathrm{CIII}] \lambda 1909$ diagrams (Fig. 5b) only if contamination by a dense region is assumed.

We find, in good agreement with De Breuck et al. (2000), that the CIII] $\lambda 1909 /[\mathrm{CII}] \lambda 2326$ ratio is a good discriminant between shocks and AGN photoionization. This ratio is plotted in Fig. 5c as a function of CIV $\lambda 1549 /[\mathrm{CII}] \lambda 2326$. Once more, two-component models appear to be the only way to account for the observations. The $n_{\mathrm{H}}=10^{6} \mathrm{~cm}^{-3}$ model is not plotted in Fig. 5c, since neither CIV $\lambda 1549 /[\mathrm{CII}] \lambda 2326$ nor CIII $] \lambda 1909 /[\mathrm{CII}] \lambda 2326$ is sensitive to density.

Figure $5 \mathrm{~d}$ shows the results of two-component models for NV $\lambda 1240 / \mathrm{HeII} \lambda 1640$ vs. NV $\lambda 1240 / \mathrm{CIV} \lambda 1549$. This diagram is often used as a metallicity diagnostic in AGN environments (Hamann \& Ferland 1993). The high NV $\lambda 1240 / \mathrm{HeII} \lambda 1640$ and NV $\lambda 1240 / \mathrm{CIV} \lambda 1549$ ratios in radio galaxies and quasars are frequently interpreted as evidence of largely super-solar metallicities, not only in 


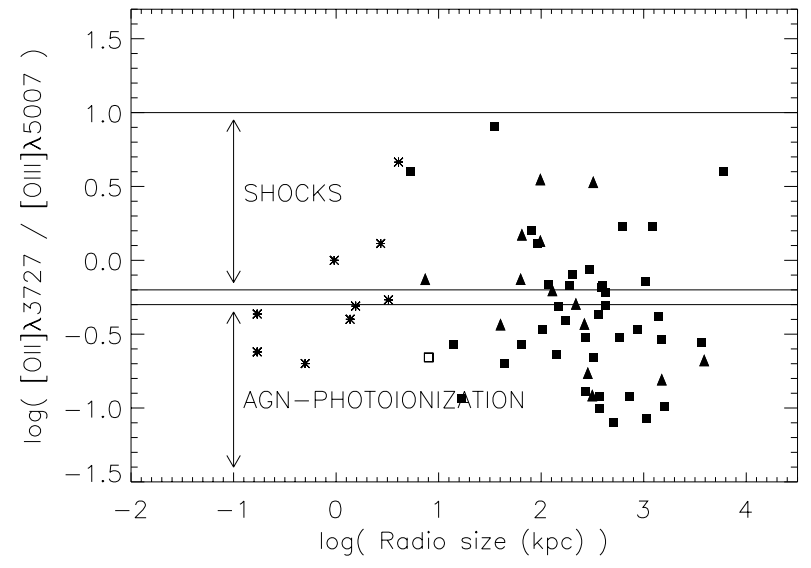

Fig. 6. Evolution of the ionization-level sensitive [OII]/[OIII] ratio as a function of the radio size in radio galaxies. Filled squares represent FRII sources and open squares FRI sources while stars represent CSS sources. Triangles are the radio galaxies without known classification. The horizontal lines show the ranges of variation of the $[\mathrm{OII}] /[\mathrm{OIII}]$ ratio in the cases of pure shocks and pure photoionization by the AGN. An interpretation of this diagram is given in Sects. 5.2 and 5.3.

broad line regions (Hamann \& Ferland 1999), but also on much larger scales (Villar-Martín et al. 1999b; De Breuck et al. 2000). Figure 5d illustrates that extreme metallicity effects are not necessarily needed to explain the intensity of the NV line. Many data points can be accounted for by $\mathrm{A}+\mathrm{S}$ and $\mathrm{A}+\mathrm{SP}$ models at solar metallicity. The objects showing the most extreme NV $\lambda 1240 / C I V \lambda 1549$ ratios can easily be accounted for by slightly increasing the metallicity $\left(Z=1.5 Z_{\odot}\right)$ in $\mathrm{A}+\mathrm{S}$ models (dotted line).

\section{Discussion}

\subsection{Evidence for ionizing shocks in extended regions}

An extensive analysis of UV and optical line ratios in extragalactic radio sources has been presented in the previous sections. The results strongly support the hypothesis that the ionized gas in radio galaxies and QSOs is excited partly by the radiation from the central source and partly by shocks. First, the model sequences obtained by varying the shock contribution to the $\mathrm{H} \beta$ flux $\left(A_{\mathrm{A} / \mathrm{S}}\right)$ between 0 and $100 \%$ provide better fits to the data than classical $U$ sequences in all line ratio diagrams. Moreover, the observational sequence from high-ionization to lowionization radio sources is naturally accounted for by varying $A_{\mathrm{A} / \mathrm{s}}$. The shock velocity lies between $100 \mathrm{~km} \mathrm{~s}^{-1}$ and $1000 \mathrm{~km} \mathrm{~s}^{-1}$. The ionization parameter $\log U$ of the photoionized clouds is typically high, $\sim-1$.

Our basic hypothesis (Sect. 1) was that ionizing shocks lie far from the nucleus inside radio sources. This assumption is a posteriori justified by the high $[\mathrm{OIII}] \lambda 5007 / \mathrm{H} \beta$ ratios observed in all the central regions (Fig. 1), and by the fact that evidence for shocks is found mainly in extended regions. Another indirect indication of the prevalence of shocks far from the nucleus are the large $\operatorname{HeII} \lambda 4686 / \mathrm{H} \beta$ ratios observed in the external parts of radio galaxies
(Fig. 4). These ratios can be accounted for only by models including a truncated (or "matter-bounded") precursor. The existence of high- $U$ MB clouds photoionized by the AGN (Binette et al. 1996) is unlikely so far from the central engine. In contrast, the hypothesis of MB precursors is reasonable. The ionizing photon source (the cooling post-shock medium) is very close. At large distances from the nucleus, it is possible that there is not enough material to absorb all the ionizing photons emitted by the post-shock zone. Finally, the hardness of the upward shock spectrum itself does not favor $100 \%$ absorption of the ionizing photons.

\subsection{The radio size-ionizing mechanism relation}

From Figs. 1 to 5 , it is clear that the balance between shocks and AGN photoionization differs from one object to another. Recently, Best et al. (2000b) presented convincing evidence that radio galaxies with radio sizes $(D)$ larger than $\sim 150 \mathrm{kpc}$ are dominated by AGN photoionization, and smaller sources by shocks. These results are based on the decrease of the $[\mathrm{CII}] \lambda 2326 / \mathrm{CIII}] \lambda 1909$ ratio at large radio size, which has been confirmed by De Breuck et al. (2000). A confirmation of the Best et al. (2000b) conclusions is clearly needed in the optical. The ionization-level sensitive $[\mathrm{OII}] \lambda 3727 /[\mathrm{OIII}] \lambda 5007$ ratio is ideal for such an analysis. The $[\mathrm{OII}] \lambda 3727 /[\mathrm{OIII}] \lambda 5007$ predictions are higher for shock models than for photoionization models (Fig. 3). In addition, [OII] and [OIII] fluxes can easily be found from the literature, for objects covering a wide range of radio sizes, permitting the construction of an ionization level-radio size diagram covering four decades in $D$. We plot in Fig. 6 the comparison between [OII]/[OIII] and radio size for our sample. For comparison with Best et al. (2000b) and De Breuck et al. (2000), as well as for the sake of homogeneity, only the integrated data for radio galaxies, both FRI/II and CSS, are plotted. Note that we have $[\mathrm{OII}]$ and $[\mathrm{OIII}]$ fluxes for 9 CSSs (compared to the 23 CSSs included in our sample). The so-called "weak line radio galaxies" (e.g. Tadhunter et al. 1998) are not plotted, due to uncertainty about the impact of the AGN inside these objects. Figure 6 also shows the ranges of $[\mathrm{OII}] /[\mathrm{OIII}]$ covered by photoionization models with $U \geq 10^{-3}$, and by shock or shock + precursor models with $100 \mathrm{~km} \mathrm{~s}^{-1} \leq v \leq 1000 \mathrm{~km} \mathrm{~s}^{-1}$.

Figure 6 confirms that there is a strong relation between the radio size and the ionization level of the gas. The most striking feature in Fig. 6 is the trend defined by CSS sources, namely a correlation between $[\mathrm{OII}] /[\mathrm{OIII}]$ and $D$, although an inverse correlation is expected if shocks dominate in the most compact sources. Large reddening effects may explain this relation if the degree of reddening is a function of radio size. Such a link is very unlikely, as demonstrated by Best et al. (2000b). If CSS sources are frustrated by a very dense medium (van Breugel et al. 1984), collisional de-excitation effects could explain the low $[\mathrm{OII}] /[\mathrm{OIII}]$ in the CSS galaxies since the critical density is lower for $[\mathrm{OII}]$ than for [OIII]. In principle, 
this could also explain the correlation between $D$ and $[\mathrm{OII}] /[\mathrm{OIII}]$ if the most compact sources are confined by the densest media.

To date, however, there is more support for the hypothesis that CSS are young rather than frustrated (de Vries et al. 1998; Owsianik \& Conway 1998; Fanti et al. 2000; Snellen et al. 2000). If it is the case, Fig. 6 strongly suggests that small radio sources are dominated by photoionization, then by shocks when they have grown up to $\sim 1 \mathrm{kpc}$, and finally by photoionization when $D \geq$ $\sim 150 \mathrm{kpc}$. One exception to this scheme is 3C 236. This galaxy, the largest FRII known $(D \sim 6 \mathrm{Mpc})$, has a high $[\mathrm{OII}] /[\mathrm{OIII}]$ ratio. This is possibly due to the existence inside $3 \mathrm{C} 236$ of an inner, compact $(2 \mathrm{kpc})$ radio source (O'Dea et al. 2001). This source, presumably shock-dominated, may dominate the integrated emission of $3 \mathrm{C} 236$.

\subsection{Why are large and compact sources photoionization-dominated?}

We now investigate the origin of the relation between radio size and shock-photoionization balance. From Fig. 6, compact/young sources seem to be dominated by photoionization. The shock contribution then increases with radio size. This may be related to the increase of the cocoon surface (and hence of the shock area) as the hot spot advances, while the photoionization component remains constant. Best et al. (2000b) interpret the prevalence of photoionization at larger radio sizes as a consequence of the shock front having passed beyond the material surrounding the AGN. Since most sources with $D \geq 200 \mathrm{kpc}$ are photoionization-dominated, this would imply that the warm gas around AGN typically expands to a radius of $\sim 100 \mathrm{kpc}$ from the nucleus.

These hypotheses about the increasing importance of ionizing shocks with time in small-scale radio sources, and their decreasing importance at larger scales, are in very good agreement with the evolution scenario of radio emission in Gigahertz Peaked Spectrum and FRIIs recently proposed by Snellen et al. (2000).

Let us suppose that the ISM density follows $n=$ $n_{0}\left(x_{\mathrm{h}} / x_{0}\right)^{-\delta}$, with $x_{0}=1 \mathrm{kpc}$ and $n_{0}=100 \mathrm{~cm}^{-3}$. The values of $\delta$ are typically $\sim 1.5$ (Blundell et al. 1999). The $\mathrm{H} \beta$ luminosity emitted by the shocks at the radio cocoon interface (Bicknell et al. 1997) depends on $v_{3}=v / 1000 \mathrm{~km} \mathrm{~s}^{-1}$, and on the shock area $A_{\mathrm{sh}}$ as:

$L_{\mathrm{H} \beta}^{\mathrm{sh}}=1.91 \times 10^{-3} v_{3}^{2.41} n A_{\mathrm{sh}}$.

The shock velocity $v$ depends on the hot spot-nucleus separation $x_{\mathrm{h}}$ as:

$v=v_{0}\left(\frac{x_{\mathrm{h}}}{x_{0}}\right)^{(\delta-2) / 3}$

where $v_{0}$ is a function of the jet energy flux $F_{\mathrm{E}}$ and of the density $n_{0}$ following $v_{0} \propto\left(F_{\mathrm{E}} / n_{0}\right)^{1 / 3}$. After elimination of $A_{\mathrm{sh}}$, Bicknell et al. (1997) show that the shock luminosity $L_{\mathrm{H} \beta}^{\mathrm{sh}}$ is given by:

$$
\begin{aligned}
L_{\mathrm{H} \beta}^{\mathrm{sh}}= & 6.7 \times 10^{41}\left(\frac{6}{8-\delta}\right)^{0.8} \\
& \times F_{\mathrm{E}}^{0.8} n^{0.2}\left(\frac{x_{\mathrm{h}}}{x_{0}}\right)^{-(\delta-2) / 5} .
\end{aligned}
$$

With $\delta \sim 1.5$, the luminosity $L_{\mathrm{H} \beta}^{\mathrm{sh}}$ increases only slowly as the hot spot advances. In this case the expansion of the radio cocoon alone is insufficient to account for the $[\mathrm{OII}] /[\mathrm{OIII}]$-radio size relation observed in CSSs. To solve this problem, the photoionization contribution may progressively decrease during the expansion, for example due to the sweeping-out of material by the radio jet.

\section{Summary and conclusions}

In this paper we have presented strong evidence that both shocks and AGN photoionization contribute to the ionization of the extended gas in radio sources. In "classical" optical line ratio diagrams, a variation between 0 and $100 \%$ of the shock contribution to the line emission mimics a variation of the ionization parameter of the gas, but the fit is better than with pure photoionization models. For [OI] $\lambda 6300 /[\mathrm{OIII}] \lambda 5007$ vs. $[\mathrm{OIII}] \lambda 4363 /[\mathrm{OIII}] \lambda 5007, \quad[\mathrm{OIII}] \lambda 5007 / \mathrm{H} \beta \quad$ vs. $[\mathrm{OIII}] \lambda 4363 /[\mathrm{OIII}] \lambda 5007$ and $\mathrm{CIII}] \lambda 1909 /[\mathrm{CII}] \lambda 2326$ vs. CIV $\lambda 1549 /[\mathrm{CII}] \lambda 2326$, the data can be reproduced only with a mixture of shocks and AGN photoionization. The $[\mathrm{OII}] /[\mathrm{OIII}]$ vs. radio size diagram shows that the most compact sources $(D \leq 2 \mathrm{kpc})$ and the sources larger than $D \sim 150 \mathrm{kpc}$ are dominated by AGN photoionization. Shocks dominate the ionizing process at intermediate radio sizes. This extends to small sizes the results of Best et al. (2000b) and De Breuck et al. (2001) obtained from UV line ratios.

These results are in good agreement with the hypothesis that the radio cocoon and hot spots lead ionizing shocks into the ambient ISM. At the beginning of the expansion phase, the shock working surface is small and AGN photoionization prevails. Then shocks become progressively important as the working surface increases and/or the amount of material swept out by the jet reduces the importance of the photoionized component. Eventually the shock front passed beyond the edge of the ISM surrounding the nucleus and AGN photoionization prevails in later phases.

High spatial resolution data are required to identify the shock-dominated zones inside radio sources and confirm our results. Integral field spectrographs are clearly the ideal instruments for this purpose, mainly when mounted on 10-m class telescopes like the VLT.

Acknowledgements. We would like to thank M. Dopita and L. Kewley for their help with using the MAPPINGSIII code, C. O'Dea for useful discussions about the relation between the radio size and the dominant ionizing mechanism in AGN, and the anonymous referee for useful suggestions and comments. EM acknowledges support of the EU TMR Network "Probing the Origin of the Extragalactic Background Radiation". 


\section{Appendix A: Source list}

Table A.1. Column 1: reference for line fluxes. Column 2: type(s) of object included in this source (RG: radio galaxies; CSS: compact steep spectrum sources; Sey: Seyfert galaxies). Column 3: reference(s) for radio size. Column 4: spatial covering. (a): reference for emission lines and radio sizes are identical. (b): 3CR atlas on line: http://www.jb.man.ac.uk/atlas/basic.html. (c): source of the [OI] 6300 flux in F10214+4724 (Lacy et al. 1998b). (d): sum of the Nucleus and EELR components. (e): sum of the Center and Tail components. (f): sum of the "a" and "c" components. (g): radio sizes estimated from the radio maps.

\begin{tabular}{|c|c|c|c|}
\hline Reference $^{1}$ & Objects types ${ }^{2}$ & Ref. for radio sizes ${ }^{3}$ & Spatial covering \\
\hline Fosbury et al. (1987) & CSS & Tadhunter et al. (1994b) & Integrated \\
\hline Robinson et al. (1987) & RG & none & Nucleus \& Extended \\
\hline Saunders et al. (1989) & $\mathrm{RG}$ & $3 \mathrm{CR}$ atlas $\mathrm{b}$ & Integrated \\
\hline McCarthy et al. (1990) & RG & $\mathrm{a}$ & Integrated \\
\hline Gelderman \& Whittle (1994) & RG, CSS, QSO & a & Integrated \\
\hline Tadhunter et al. (1994a) & RG & Krichbaum et al. (1998) & Extended \\
\hline Soifer et al. $(1995)^{\mathrm{c}}$ & QSO & none & Integrated \\
\hline Simpson et al. (1996) & RG & none & Integrated \\
\hline Dey et al. (1997) & RG & none & Integrated \\
\hline Morganti et al. (1997) & CSS & a & Integrated \\
\hline Röttgering et al. (1997) & RG & Röttgering et al. (1994) & Integrated \\
\hline Clark et al. $(1998)^{\mathrm{d}}$ & $\mathrm{RG}$ & Baum et al. (1988) & Integrated \\
\hline Koekemoer \& Bicknell (1998) & RG & Subrahmanyan et al. (1996) & Integrated \\
\hline Lacy et al. $(1998 a)^{f}$ & RG & none & Integrated \\
\hline Lacy et al. (1998b) & QSO & Lawrence et al. (1993) & Integrated \\
\hline Tadhunter et al. (1998) & RG, CSS, QSO & Morganti et al. $(1993)^{\mathrm{g}}$ & Integrated \\
\hline Villar-Martín et al. (1998) & RG & $\mathrm{a}$ & Integrated \\
\hline Best et al. (1999) & RG & a & Integrated \\
\hline Lacy et al. (1999) & $\mathrm{RG}, \mathrm{QSO}$ & $\mathrm{a}$ & Integrated \\
\hline Murayama et al. (1999) & QSO & none & Integrated \\
\hline Simpson et al. (1999) & $\mathrm{RG}$ & $\mathrm{a}$ & Integrated \\
\hline Snellen et al. (1999) & $\mathrm{RG}, \mathrm{QSO}$ & none & Integrated \\
\hline Villar-Martín et al. (1999b) & RG & $\begin{array}{l}\text { Pentericci et al. (1999), } \\
\text { Röttgering et al. (1994) }\end{array}$ & Integrated \\
\hline Villar-Martín et al. (1999c) & $\mathrm{RG}$ & Morganti et al. (1993) & Extended \\
\hline Best et al. (2000a) & RG & $\mathrm{a}$ & Integrated \\
\hline De Breuck et al. (2000) & $\mathrm{RG}$ & $\mathrm{a}$ & Integrated \\
\hline Palma et al. (2000) & RG & $\mathrm{a}$ & Integrated \\
\hline Schoenmakers (2000) & $\mathrm{RG}$ & $\mathrm{a}$ & Integrated \\
\hline De Breuck et al. (2001) & RG & $\mathrm{a}$ & Integrated \\
\hline
\end{tabular}




\section{References}

Aldrovandi, S. M. V., \& Contini, M. 1984, A\&A, 140, 368 Aldrovandi S. M. V., \& Contini, M. 1985, A\&A, 149, 109

Antonucci, R. 1993, ARA\&A, 31, 473

Baldwin, J. A., Phillips, M. M., \& Terlevich, R. 1981, PASP, 93,19

Baum, S. A., \& Heckmann, T. 1989, ApJ, 336, 702

Baum, S. A., Heckmann, T., Bridle, A., van Breugel, W., \& Miley, G. 1988, ApJS, 68, 643

Best, P. N., Röttgering, H. J. A., \& Lehnert, M. D. 1999, MNRAS, 310, 223

Best, P. N., Röttgering, H. J. A., \& Longair, M. S. 2000a, MNRAS, 311, 1

Best, P. N., Röttgering, H. J. A., \& Longair, M. S. 2000b, MNRAS, 311, 23

Bicknell, G. V., Dopita, M. A., \& O'Dea, C. P. O. 1997, ApJ, 485, 112

Binette, L., Wilson, A. S., \& Storchi-Bergmann, T. 1996, A\&A, 312,365

Blundell, K. M., Rawlings, S., \& Willott, C. J. 1999, AJ, 117, 677

Bresolin, F., Kennicutt, R. C., \& Garnett, D. R. 1999, ApJ, 510, 104

Clark, N. E., Axon, D. J., Tadhunter, C. N., Robinson, A., \& O'Brien, P. 1998, ApJ, 494, 546

Contini, M., \& Aldrovandi, S. M. V. 1983, A\&A, 127, 15

Contini, M., \& Aldrovandi, S. M. V. 1986, A\&A, 168, 41

Contini, M., \& Viegas-Aldrovandi, S. M. 1987, A\&A, 185, 39

de Breuck, C., Röttgering, H., Miley, G., van Breugel, W., \& Best, P. 2000, A\&A, 362, 519

de Breuck, C., van Breugel, W., Röttgering, H., et al. 2001, AJ, 121, 1241

De Vries, W. H., O’Dea, C. P., Baum, S. A., et al. 1998, ApJ, 503,156

Dopita, M. A., \& Sutherland, R. S. 1995, ApJ, 455, 468

Dopita, M. A., \& Sutherland, R. S. 1996, ApJS, 102, 161

Durret, F. 1990, A\&A, 229, 351

Fanaroff, B. L., \& Riley, J. M. 1974, MNRAS, 167, 31

Fanti, C., Pozzi, F., Fanti, R., et al. 2000, A\&A, 358, 499

Ferland, G. J. 1996, HAZY, a brief introduction to Cloudy, University of Kentucky, Department of Physics and Astronomy Internal Report

Ferland, G. J., \& Osterbrock, D. E. 1987, ApJ, 318, 145

Gelderman, R., \& Whittle, M. 1994, ApJS, 91, 491

Hamann, F., \& Ferland, G. 1993, ApJ, 418, 11

Hamann, F., \& Ferland, G. 1999, ARA\&A, 37, 487

Hes, R., Barthel, P. D., \& Fosbury, R. A. E. 1996, A\&A, 313, 423

Koekemoer, A. M., \& Bicknell, G. V. 1998, ApJ, 497, 662

Koski, A. T., \& Osterbrock, D. E. 1976, ApJ, 203, L49

Krichbaum, T. P., Alef, W., Witzel, A., et al. 1998, A\&A, 329, 873

Lacy, M., Rawlings, S., Blundell, K. M., \& Ridgway, S. E. 1998a, MNRAS, 298, 966

Lacy, M., Rawlings, S., \& Serjeant, S. 1998b, MNRAS, 299, 1220

Lacy, M., Rawlings, S., Hill, G. J., et al. 1999, MNRAS, 308, 1096

Lehnert, M. D., Miley, G. K., Sparks, W. B., et al. 1999, ApJS, 123,351

McCarthy, P. J., Spinrad, H., Dickinson, M., et al. 1990, ApJ, 365,487
McCarthy, P. J., Spinrad, H., \& van Breugel, W. 1995, ApJS, 99, 27

Morganti, R., Killeen, N. E. B., \& Tadhunter, C. N. 1993, MNRAS, 263, 1023

Morganti, R., Tadhunter, C. N., Dickson, R., \& Shaw, M. 1997, A\&A, 326, 130

Moy, E. 2000, Ph.D. Thesis, Institut d'Astrophysique de Paris

Moy, E., Rocca-Volmerange, B., \& Fioc, M. 2001, A\&A, 365, 347

Murayama, T., Taniguchi, Y., Evans, A. S., et al. 1999, AJ, 117,1645

Owsianik, I., \& Conway, J. E. 1998, A\&A, 69, 337

Palma, C., Bauer, F. E., Cotton, W. D., et al. 2000, AJ, 119, 2068

Pentericci, L., Röttgering, H. J. A., Miley, G. K., et al. 1999, A\&A, 341, 329

Prieto, M. A., Walsh, J. R., Fosbury, R. A. E., \& di Serego Alighieri, S. 1993, MNRAS, 263, 10

Ridgway, S. E., \& Stockton, A. 1997, AJ, 114, 511

Robinson, A., Binette, L., Fosbury, R. A. E., \& Tadhunter, C. N. 1987, MNRAS, 227, 97

Röttgering, H. J. A., Lacy, M., Miley, G. K., Chambers, K. C., \& Saunders, R. 1994, A\&A, 108, 79

Röttgering, H. J. A., van Ojik, R., Mileu, G. K., et al. 1997, A\&A, 326, 505

Saunders, R., Baldwin, J. E., Rawlings, S., Warner, P. J., \& Miller, L. 1989, MNRAS, 238, 777

Schoenmakers, A. P. 2000, Ph.D. Thesis, Universiteit Leiden

Simpson, C., Eisenhardt, P., Armus, L., et al. 1999, ApJ, 525, 659

Simpson, C., Ward, M., Clements, D. L., \& Rawlings, S. 1996, MNRAS, 281, 509

Snellen, I. A. G., Schilizzi, R. T., Bremer, M. N., et al. 1999, MNRAS, 307, 149

Snellen, I. A. G., Schilizzi, R. T., Miley, G. K., et al. 2000, MNRAS, 319, 445

Soifer, B. T., Cohen, J. G., Armus, L., et al. 1995, ApJ, 443, L65

Spinrad, H., \& Djorgovski, S. 1984, ApJ, 285, L49

Subrahmanyan, R., Saripalli, L., \& Hunstead, R. W. 1996, MNRAS, 279, 257

Sutherland, R. S., Bicknell, G. V., \& Dopita, M. A. 1993, ApJ, 414,510

Tadhunter, C. N., Fosbury, R. A. E., di Serego Alighieri, S., et al. 1988, MNRAS, 235, 403

Tadhunter, C. N., Metz, S., \& Robinson, A. 1994a, MNRAS, 268,989

Tadhunter, C. N., Shaw, M. A., \& Morganti, R. 1994b, MNRAS, 271, 807

Tadhunter, C. N., Morganti, R., Robinson, A., et al. 1998, MNRAS, 298, 1035

van Breugel, W. J. M., Heckman, T., \& Miley, G. K. 1984, AJ, 89,5

Viegas-Aldrovandi, S. M., \& Contini, M. 1989, A\&A, 215, 253 Villar-Martín, M., Binette, L., \& Fosbury, R. A. E. 1999a, A\&A, 346, 12

Villar-Martín, M., Fosbury, R. A. E., Binette, L., Tadhunter, C. N., \& Rocca-Volmerange, B. 1999b, A\&A, 351, 47

Villar-Martín, M., Tadhunter, C., Morganti, R., Axon, D., \& Koekemoer, A. 1999c, MNRAS, 307, 24

Villar-Martín, M., Tadhunter, C., \& Clark, N. 1997, A\&A, 323, 21

Villar-Martín, M., Tadhunter, C., Morganti, R., et al. 1998, A\&A, 332, 479 